\title{
Multiphoton Imaging of Porcine Eye: Cornea, Limbus, Conjunctiva, and Scelera
}

\author{
Shu-Wen Teng ${ }^{1}$, Ju-Li Peng ${ }^{2}$, Huei-Hsing Lin ${ }^{2}$, Hai-Yin $\mathrm{Wu}^{1}$, Wen $\mathrm{Lo}^{1}$, Yen Sun ${ }^{1}$, \\ Wei-Chou Lin ${ }^{3}$, Sung-Jan Lin ${ }^{4}$, Shiou-Hwa Jee ${ }^{5}$, Hsin-Yuan Tan ${ }^{6}$, \\ Chen-Yuan Dong ${ }^{1}$ * \\ ${ }^{1}$ Department of Physics, National Taiwan University, Taipei 106, Taiwan \\ ${ }^{2}$ Department of Life Science, National Taiwan University, Taipei 106, Taiwan \\ ${ }^{3}$ Department of Pathology, National Taiwan University Hospital, Taipei 100, Taiwan \\ ${ }^{4}$ Department of Dermatology, National Taiwan University Hospital, Taipei 100, \\ Taiwan \\ ${ }^{5}$ Department of Dermatology, College of Medicine, National Taiwan University \\ Hospital, Taipei 100, Taiwan \\ ${ }^{6}$ Department of Ophthalmology, Chang Gung Memorial Hospital, Linko 333, Taiwan
}

*To whom correspondence should be addressed : cydong@phys.ntu.edu.tw

Tel.: 886-2-3366-5155 ; Fax : 886-2-3366-5244

Abstract:This purpose of this study is to demonstrate the feasibility of using multiphoton microscopy in imaging eye surface. Specifically, the cornea, limbus, conjunctiva, and sclera were imaged using multiphoton induced fluorescence and second-harmonic generation (SHG) imaging.

\section{INTRODUCTIONS}

Without extrinsic fluorescent molecules, we were able to image different parts of the eye surface. Our results show that individual corneal epithelial cells (autofluorescence) are visible as are the well organized comeal fibers (SHG). The histological mo rphology of the limbus also correlates well with the multiphoton image. With increasing depths, multiphoton limbus imaging reveals the surface epithelial cells followed by joining of the corneal and scelera collagen fibers. The conjunctiva and sclera are also visible under multiphoton excitation.

\section{METHODS AND RESULTS}

Shown in Fig. 1 is the multiphoton microscope used in this study. Figs. 2(a),2(b) are, respectively, large area multiphoton images of the limbus, detailed image of the limbus. Autfluorescence (green) and second harmonic generation (blue) signals can be used to identify morphological features of the limbus and sclera.
Figuar I

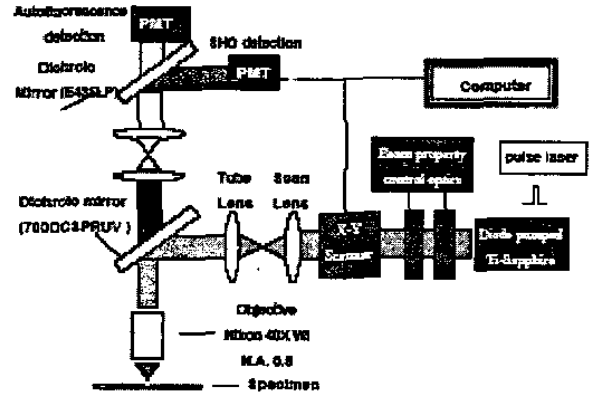




\section{THP-28(T5)}

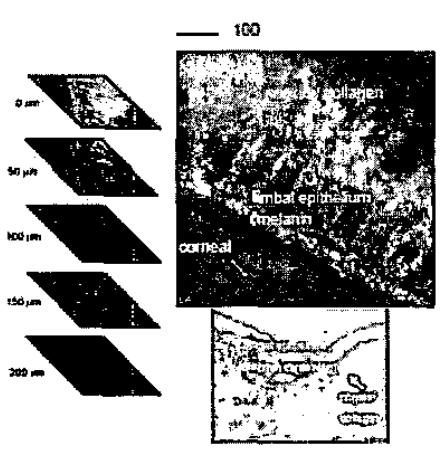

Figure $2(a)$

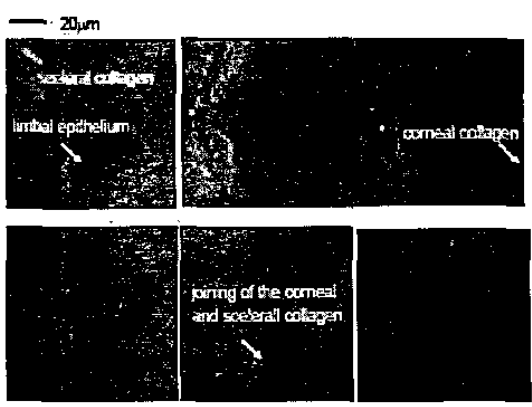

Figure 2 (b)

\section{CONCLUSIONS}

Our demonstrate that without the introduction of extrinsic fluorescent molecules, multiphoton induced fluorescence and SHG signals can be used to obtain structural information of intact porcine eyes. Autofluorescence from the epithelia and SHG from collagen fibers can be acquired for structural analysis of intact eyes. We have demonstrated that with additional developments, multiphoton microscopy may be used for in vivo investigation of ophthalmologic disease diagnosis and tissue physiological studies.

\section{REFERENCES}

1. Ross MH, Kaye GI, Pawlina W. Histology. 4th ed. Baltimore. MD: Lippincott Williams \& Wilkins; 2003: 788-810.

2. Nassif NA, Cense B, Park BH, Pierce MC, Yun SH, Bouma BE, Tearney GJ,Chen TC, de Boer JF. 2004. In vivo high-resolution video-rate spectral-domain optical coherence tomography of the human retina and optic nerve. Optics Express 12(3):367-376.

3. Romano AC, Espana EM, Yoo SH, Budak MT, Wolosin JM, Tseng SCG. 2003.Different cell sizes in human limbal and central corneal basal epithelia measuredby confocal microscopy and flow cytometry. Investigative Ophthalmology \&Visual Science 44(12):5125-5129.

4. Denk W, Strickler JH, Webb WW. 2-photon laser scanning fluorescence microscopy. Science. 1990; 248(4951): 73-76.

5. So PTC, Dong CY, Masters BR, Berland KM. Two-photon excitation fluorescence microscopy. Annual Review of Biomedical Engineering. 2000; 2:399-429.

6. Denk W, Sugimori M, Llinas R. 2 types of calcium response limited to single spines in cerebellar Purkinje-cells. Proceedings of the National Academy of Sciences of the United States of America. 1995; 92(18): 8279-8282.

$$
\text { ( }
$$

7. Squirrell JM, Wokosin DL, White JG, Bavister BD. Long-term two-photon fluorescence imaging of mammalian embryos without compromising viability. Nature Biotechnology. 1999; 17(8): 763-767.

8. So PTC, Kim H, Kochevar IE. Two-photon deep tissue ex vivo imaging of mouse dermal and subcutaneous structures. Optics Express. 1998;3(9), 339-350.

9. Miller MJ, Wei SH, Parker I, Cahalan MD. Two-photon imaging of lymphocyte motility and antigen response in intact lymph node. Science.2002; 296(5574):1869-1873.

10. Brown E, McKee T, diTomaso E, Pluen A, Seed B, Boucher Y, Jain RK. Dynamic imaging of collagen and its modulation in tumors in vivo using second-harmonic generation. Nature Medicine. 2003; $9(6): 796-800$. 